\title{
FENOLOGÍA Y PRODUCTIVIDAD DEL AGUA EN VARIEDADES MEJORADAS DE FRIJOL PINTO CULTIVADAS EN DURANGO, MÉXICO
}

\author{
PHENOLOGY AND WATER PRODUCTIVITY IN IMPROVED PINTO \\ BEAN VARIETIES GROWN IN DURANGO, MEXICO
}

\author{
Rigoberto Rosales-Serna ${ }^{1}$, Hilario Flores-Gallardo ${ }^{1,2 *}$, Juan C. López- \\ González ${ }^{3}$, Juan E. Rubiños-Panta ${ }^{2}$, Ixchel A. Ortiz-Sánchez ${ }^{3}$, Héctor Flores- \\ Magdaleno ${ }^{2}$, Saúl Santana-Espinoza' y Pablo A. Domínguez-Martínez ${ }^{1}$
}

\begin{abstract}
'Instituto Nacional de Investigaciones Forestales, Agrícolas y Pecuarias (INIFAP), Campo Experimental Valle del Guadiana, Durango, México. ${ }^{2}$ Colegio de Postgraduados, Programa de Hidrociencias, Montecillo, Texcoco, Estado de México, México. ${ }^{3}$ Tecnológico Nacional de México, Instituto Tecnológico del Valle del Guadiana, Durango, México.
\end{abstract}

*Autor de correspondencia (flores.hilario@inifap.gob.mx)

\section{RESUMEN}

El agua es uno de los insumos más importantes en la producción de frijol en el estado de Durango, México; las nuevas variedades mejoradas de frijol no están completamente caracterizadas por su respuesta a este factor, lo cual es necesario realizar con fines de tecnificación y planeación del riego, así como para la predicción de la fenología y mejorar la productividad del agua. El objetivo del presente estudio fue evaluar la fenología con la estimación de grados día ( ${ }^{\circ} D$ ) y productividad del agua (PA) en dos variedades mejoradas de frijol, bajo tres niveles de humedad con riego por gravedad, cultivadas en Durango, México. Las variedades Pinto Saltillo y Pinto Centauro se sembraron en los ciclos primavera-verano (P-V) de 2015 y 2016 en Durango y en P-V 2015 en Canatlán. El diseño experimental fue bloques completos al azar con cuatro repeticiones en 2015 y ocho repeticiones en 2016. La parcela experimental consistió de 12 a 32 surcos de 8 a $10 \mathrm{~m}$ de longitud y $0.81 \mathrm{~m}$ de separación. Se aplicaron tres niveles de humedad que consistieron en riego total (RT: $100 \%$ de capacidad de campo) y riego deficitario (RD: 80 y $60 \%$ con respecto a RT). Se registró la lámina de riego $(\mathrm{Lr})$, ${ }^{\circ} \mathrm{D}$, días después de la siembra (dds), fenología, rendimiento de grano y PA. Se realizó un análisis de varianza y comparación de medias con la prueba de Tukey $(P \leq 0.05)$. Con relación a los dds y ${ }^{\circ} D$, Pinto Centauro mostró precocidad (floración $40_{406}$ a $41_{430}$ y madurez $92_{893}$ a $99_{937} \mathrm{dds}_{{ }_{\circ}}$ ) con respecto a Pinto Saltillo (floración $42_{438}$ a $45_{459}$ y madurez $93_{900}$ a $103_{1013}$ dds $_{\circ \mathrm{D}}$ ). El rendimiento de grano no mostró diferencias significativas en Durango, pero en Canatlán se observaron diferencias significativas entre niveles de humedad y variedades. La PA varió entre 3.3 y $8.0 \mathrm{~kg} \mathrm{ha}^{-1} \mathrm{~mm}^{-1}$, lo cual influyó en la fluctuación del rendimiento entre 2546 y $3687 \mathrm{~kg} \mathrm{ha}^{-1}$.

Palabras clave: Phaseolus vulgaris, rendimiento de grano, riego, sostenibilidad.

\section{SUMMARY}

Water is one of the most important inputs in bean production in the state of Durango, Mexico; the new improved bean varieties are not fully characterized by their response to this factor, which is necessary for the purposes of modernization and irrigation planning, as well as for the prediction of phenology, to improve water productivity. The objective of this study was to evaluate phenology with the estimation of degree days $\left({ }^{\circ} \mathrm{D}\right)$ and water productivity (WP) in two improved bean varieties, under three levels of moisture by gravity irrigation, grown in Durango, Mexico. Pinto Saltillo and Pinto Centauro varieties were sown in the Spring-Summer season (SS) of
2015 and 2016 in Durango and in SS 2015 in Canatlán. The experimental design was randomized complete blocks with four replications in 2015 and eight replications in 2016. The experimental plot consisted of 12 to 32 rows of 8 to $10 \mathrm{~m}$ in length and $0.81 \mathrm{~m}$ apart. Three levels of moisture were applied consisting of total irrigation (TI: $100 \%$ field capacity) and deficit irrigation (DI: 80 and $60 \%$ with respect to $\mathrm{TI}$ ). The irrigation sheet (Is), ${ }^{\circ} \mathrm{D}$, days after sowing (das), phenology, grain yield and WP were registered. Analysis of variance and Tukey test $(P \leq 0.05)$ were performed. Regarding the das and ${ }^{\circ} D$, Pinto Centauro showed earliness (flowering $40_{406}$ to $41_{430}$ and maturity $92_{893}$ to $99_{937}$ das $_{{ }_{0}}$ ) compared to Pinto Saltillo (flowering $42_{438}$ to $45_{459}$ and maturity $93_{900}$ to $103_{1013}$ das $_{{ }_{0}}$ ). Grain yield did not show significant differences in Durango but in Canatlán significant differences were observed between moisture levels and varieties. The WP ranged from 3.3 to $8.0 \mathrm{~kg} \mathrm{ha}^{-1} \mathrm{~mm}^{-1}$, which influenced the fluctuation in yield between 2546 and $3687 \mathrm{~kg} \mathrm{ha}^{-1}$.

Index words: Phaseolus vulgaris, grain yield, irrigation, sustainability

\section{INTRODUCCIÓN}

El grano de frijol (Phaseolus vulgaris) es un producto básico en México debido a que luego de su cocción es fuente de proteínas en el consumo humano. Esta leguminosa es de gran importancia económica, nutricia y nutracéutica, sin embargo, se cultiva principalmente en condiciones de secano (Guzmán et al., 2002; RíosUglade et al., 2006; Rosales-Serna et al. 2016), donde el estrés intermitente ocasionado por insuficiencia de humedad reduce el rendimiento potencial de grano $y_{\text {, en }}$ consecuencia, afecta negativamente la economía del agricultor (Rosales-Serna et al., 2004).

De acuerdo con Borja-Bravo et al. (2018), el altiplano semiárido del centro-norte de México es una región que abarca parte de las entidades de Chihuahua, Coahuila, Durango, Zacatecas, Aguascalientes, San Luis Potosí, Jalisco, Guanajuato y Querétaro, y se caracteriza por su clima semidesértico, esta región es la más importante en producción de frijol en México porque se cultiva dicha 
leguminosa en gran superficie bajo condiciones de temporal (secano).

En Durango, en años recientes dio inicio el cultivo de frijol bajo riego para aumentar su productividad, estabilizar el rendimiento e incrementar el valor comercial del grano (Rosales-Serna et al. 2016); asimismo, el mejoramiento genético ha generado variedades de frijol precoces y de bajo requerimiento hídrico, con las que se han logrado incrementos significativos en el rendimiento durante años con climas favorables (> $450 \mathrm{~mm}$ ) y distribución apropiada de la lluvia (Rosales-Serna et al., 2019). En este contexto, es necesario determinar la capacidad de la producción de grano y de adaptación a las condiciones ambientales de la región por parte de las nuevas variedades mejoradas de frijol (Arellano-Arciniega et al., 2015). La caracterización de las variedades sembradas, actualmente incompleta, es fundamental para la tecnificación y planeación del riego, así como para la predicción de la fenología, para mejorar la productividad del agua (PA) y rendimiento de grano (de Medeiros et al., 2016; de Oliveira et al., 2012; RosalesSerna et al. 2016).

Las variedades de frijol pinto, predominantes en siembras comerciales en Durango (Pinto Saltillo y Pinto Villa) son sensibles al fotoperiodo (Rosales et al., 2012; RosalesSerna et al., 2004; Sanchez-Valdez et al., 2004). La utilidad de este carácter es limitada en condiciones de riego, donde se prefieren variedades neutrales en su respuesta al fotoperiodo para aprovechar la posibilidad de sembrar en cualquier época del año, siempre y cuando existan temperaturas favorables para el frijol $\left(10\right.$ a $\left.28^{\circ} \mathrm{C}\right)$. Otra área de oportunidad es conocer la influencia de la temperatura sobre el desarrollo de las variedades modernas de frijol cultivadas en Durango.

Existen varios métodos para predecir la fenología del frijol en diferentes ambientes de producción (Baez-González et al., 2015); sin embargo, el concepto grados día ( $\left.{ }^{\circ} D\right)$ se basa en las temperaturas (Flores-Magdaleno et al., 2014), por lo cual, resulta uno de los métodos más precisos para estimar la acumulación de ${ }^{\circ} \mathrm{D}$ entre los umbrales térmicos para el desarrollo de cada especie (Flores-Gallardo et al., 2012; Ojeda-Bustamante et al., 2004; 2006). Por otra parte, para evaluar la PA, el método más utilizado incluye la relación entre el rendimiento obtenido y el volumen de agua aplicado, medido con base en la evapotranspiración acumulada del cultivo (ETc) y láminas de riego (Lr) (Hari Krishna et al., 2014; Rosales-Serna et al., 2016). Las condiciones favorables de humedad pueden propiciar un incremento significativo del rendimiento de grano, así como un desarrollo fenológico favorable del frijol bajo condiciones meteorológicas contrastantes; por lo tanto, el objetivo de este estudio fue evaluar la fenología en función del tiempo térmico y la PA en dos variedades mejoradas de frijol pinto cultivadas en Durango.

\section{MATERIALES Y MÉTODOS}

\section{Descripción del área de estudio}

Los trabajos de campo se realizaron en dos ambientes (Durango y Canatlán), ubicados en el Distrito de Riego 052 del estado de Durango, México. En el sitio de Durango $\left(23^{\circ} 59^{\prime} 15^{\prime \prime} \mathrm{N}, 104^{\circ} 37^{\prime} 16^{\prime \prime} \mathrm{O}\right.$ y una altitud de $1879 \mathrm{~m}$ ) se trabajó durante los ciclos agrícolas primavera-verano (P-V) 2015 y 2016; el tipo de textura del suelo es franco arenoso (Cuadro 1), con capacidad intermedia para la retención de humedad, profundidad media, pendiente de 0 a $2 \%, p H ~ 7.9$ (moderadamente alcalino) y contenido pobre de materia orgánica (1.2 \%). En el sitio de Canatlán ( $24^{\circ} 33^{\prime} 08^{\prime \prime} \mathrm{N}, 104^{\circ} 44^{\prime} 33^{\prime \prime} \mathrm{O}$ y una altitud de $\left.1945 \mathrm{~m}\right)$ el cultivo se estableció en P-V 2015. El tipo de textura del suelo es arena franca, con baja capacidad de retención de humedad, profundidad media, pendiente de 0 a $4 \%$, pH 8.3 (moderadamente alcalino) y contenido pobre de materia orgánica (1.4\%). El clima predominante en los dos sitios es templado semiárido [BS, Kw (w) (e)], con régimen de lluvias en verano y fuerte variación de la temperatura media anual con un registro de $17.4^{\circ} \mathrm{C}$ (García, 1987). La Iluvia acumulada durante el año alcanza un promedio de $476 \mathrm{~mm}$, con valores altos entre junio y septiembre.

\section{Tratamientos evaluados, diseño y unidad experimental}

Los factores y niveles estudiados fueron: variedades de frijol (Pinto Saltillo y Pinto Centauro) y niveles de humedad (3) en cada sitio y ciclo. El diseño experimental fue bloques completos al azar, con cuatro repeticiones en 2015 y ocho repeticiones en 2016. La parcela experimental fue de 32 surcos de $10 \mathrm{~m}$ de largo (Durango, 2015), 16 surcos de $8 \mathrm{~m}$ de largo (Canatlán, 2015) y 12 surcos con una longitud de $10 \mathrm{~m}$ (Durango, 2016). En todos los sitios se establecieron surcos con $0.81 \mathrm{~m}$ de separación.

Se aplicaron tres niveles de humedad del suelo mediante riego por gravedad, los cuales consistieron en: riego total, RT $100 \%$ de humedad aprovechable (HA) de humedad aprovechable (HA) para llegar a capacidad de campo (riego total, RT) y riego deficitario, RD $80 \%$ de HA y RD 60 $\%$ de $\mathrm{HA}$, que involucran un déficit de humedad en el suelo.

\section{Manejo del cultivo y monitoreo de la humedad}

Las fechas de siembra fueron el 10 de julio (Durango, 2015), 13 de julio (Canatlán, 2015) y 07 de julio (Durango, 2016) y se realizaron dos escardas, una aplicación del herbicida Flex ${ }^{\circledR}$ (Fomesafen) y dos deshierbes manuales, 
Cuadro 1. Características físicas del suelo en cada sitio de evaluación con los tres niveles de humedad sobre la fenología y PA en frijol.

\begin{tabular}{lcccccccc}
\hline Sitio & Textura & $\mathrm{A}(\%)$ & $\mathrm{L}(\%)$ & $\mathrm{R}(\%)$ & $\theta_{\mathrm{CC}}(\%)$ & $\theta_{\mathrm{PMP}}(\%)$ & $\mathrm{HA}(\%)$ & $\left.\rho_{\mathrm{a}}(\mathrm{g} \mathrm{cm})^{-3}\right)$ \\
\hline Durango & Franco arenoso & 63 & 28 & 9 & 21 & 9 & 12 & 1.2 \\
Canatlán & Arena franca & 87 & 11 & 2 & 11 & 4 & 7 & 1.4 \\
\hline
\end{tabular}

A: arena, L: limo, R: arcilla, $\theta_{\text {cc }}$ : capacidad de campo, $\theta_{\text {PMP: }}$ punto de marchitez permanente, $\mathrm{HA}$ : humedad aprovechable, $\rho_{\mathrm{a}}$ : densidad aparente del suelo.

para mantener el cultivo libre de maleza. En todos los casos, la fertilización se aplicó durante la primera escarda y se utilizó la dosis 35-50-00 $\left(\mathrm{N}-\mathrm{P}_{2} \mathrm{O}_{5}-\mathrm{K}_{2} \mathrm{O}\right)$.

El contenido de humedad se monitoreó mediante el uso de un reflectómetro (Time Domain reflectometer TDR HydroSense II, Campbell Scientific, Logan Utah, EUA). Los datos de humedad obtenidos antes y después del riego se usaron para estimar la lámina de riego aplicada $\left(L r_{a}\right)$ en cada nivel de humedad, la cual se calculó con la siguiente ecuación:

$$
L r_{a}=\left(\frac{\theta c c-W a}{100}\right) \rho_{a} \times \operatorname{Pr}
$$

donde $\theta_{\text {cc }}$ es la capacidad de campo (\%), Wa es el contenido de humedad en el suelo antes del riego (\%), $\rho_{\text {a }}$ es la densidad aparente del suelo $\left(\mathrm{g} \mathrm{cm}^{-3}\right)$ y Pr es la profundidad efectiva de las raíces $(30 \mathrm{~cm}$ ) (Rosales-Serna et al., 2016).

\section{Variables evaluadas}

El registro fenológico (desde la emergencia hasta la madurez fisiológica) incluyó el número de dds y los grados día ( $\left.{ }^{\circ} D\right)$ (Ojeda-Bustamante et al., 2004; 2006). Se registraron las variables de temperatura máxima $\left(T_{\text {máx }}\right)$ temperatura mínima $\left(T_{\text {min }}\right)$ y precipitación (Pp) de acuerdo con INIFAP (2017), así como los datos de rendimiento de grano y PA. Con la ecuación 2 se calculó la lámina de riego neta $\left(L r_{n}\right)$ en $\mathrm{mm}$ (Cuadro 2).

$$
L r_{n}=P p+r
$$

donde Pp es la precipitación registrada $(\mathrm{mm})$ y r es el riego aplicado (mm).

El número de ${ }^{\circ} D$ se estimó con las siguientes condicionantes (ecuaciones 3, 4 y 5), propuestas por Ojeda-Bustamante et al. (2004; 2006)

$$
{ }^{\circ} \mathrm{D}=T-\mathrm{T}_{\min } \text { si } T<\mathrm{T}_{\text {máx }}
$$

$$
\begin{gathered}
{ }^{\circ} \mathrm{D}=\mathrm{T}_{\text {máx }}-\mathrm{T}_{\text {min }} \text { si } T \geq T_{\text {máx }} \\
{ }^{\circ} \mathrm{D}=0 \text { si } T \leq T_{\text {min }}
\end{gathered}
$$

donde $T$ es la temperatura media diaria, $T_{\text {min }}\left(10^{\circ} \mathrm{C}\right)$ y $T_{\text {máx }}$ $\left(28^{\circ} \mathrm{C}\right)$ representan la temperatura mínima y máxima que delimitan el intervalo requerido (umbral máximo y mínimo) para el crecimiento de la planta del frijol (de Medeiros et al., 2016; Jenni et al., 2000). En madurez, se tomaron tres submuestras de plantas para determinar el rendimiento de grano y luego calcular el promedio para cada repetición. Las submuestras consistieron de dos surcos de $5 \mathrm{~m}$ de longitud, con una separación de $0.81 \mathrm{~m}\left(8.1 \mathrm{~m}^{2}\right)$. La PA se calculó con la ecuación 6 (Hari Krishna et al., 2014; Rosales-Serna et al., 2016):

$$
\mathrm{PA}=\frac{\text { rendimiento }\left(\mathrm{kg} \mathrm{ha}^{-1}\right)}{L r_{\mathrm{n}}}
$$

donde $L r_{n}$ es la lámina de riego neta $(\mathrm{mm})$ calculada con la ecuación 2.

\section{Análisis estadístico}

Se realizó un análisis de varianza para rendimiento con el modelo lineal correspondiente a un diseño de bloques completos al azar. Las medias se compararon con la prueba de Tukey $(P \leq 0.05)$. Estos análisis se realizaron con SAS ${ }^{\circledR}$ versión 9.4 (SAS Institute, 2013).

\section{RESULTADOS Y DISCUSIÓN}

\section{Rendimiento}

La variedad Pinto Centauro mostró precocidad para floración (40-41 dds) y madurez (92-99 dds) en los dos ambientes, en comparación con Pinto Saltillo (floración 42-45 dds y madurez 93 a 103 dds), mostrando diferencias significativas entre variedades (Cuadro 3). De igual forma, la acumulación de ${ }^{\circ} \mathrm{D}$ fue inferior en Pinto Centauro (floración 406 a $430{ }^{\circ} \mathrm{D}$ y madurez $893-937^{\circ} \mathrm{D}$ ) en comparación con Pinto Saltillo (floración 438 a 459 D y madurez 900-1013 
$\left.{ }^{\circ} D\right)$. La fenología contabilizada en días mostró tendencia similar y relación estrecha con la estimación realizada con base en el tiempo térmico. Los resultados muestran que es posible utilizar cualquiera de las variables que describen el desarrollo del frijol y, aunque la evaluación con base en dds es más sencilla por su determinación visual, el uso de ${ }^{\circ} D$ es común en los programas de cómputo para la predicción fenológica, por su viabilidad y precisión (OjedaBustamante et al., 2006).

El rendimiento fue estadísticamente igual entre variedades y condiciones de humedad en Durango (2015 y 2016) y únicamente en Canatlán (2015) se observaron diferencias $(P \leq 0.0001)$ entre niveles de humedad (Cuadro 3). En este último sitio se observó igualdad estadística entre variedades y el valor más alto de rendimiento en ambas variedades (Pinto Centauro $3157 \mathrm{~kg} \mathrm{ha}^{-1}$ y Pinto Saltillo $\left.3344 \mathrm{~kg} \mathrm{ha}^{-1}\right)$ se registró con el nivel de humedad al 100 $\%$ (631 mm), mientras que en el nivel de $60 \%$ de humedad
(401 mm) se registraron valores significativamente inferiores. La respuesta fue similar en ambas variedades, lo cual dependió fundamentalmente del incremento de la cantidad de agua aplicada $(631 \mathrm{~mm})$. También se deduce que la respuesta similar entre ambas variedades pudo deberse a su cercanía genética, y su potencial de rendimiento similar había sido previamente demostrado (Rosales et al., 2012).

En Durango, durante el ciclo 2015, se obtuvieron rendimientos entre 2546 y $3380 \mathrm{~kg} \mathrm{ha}^{-1}$, mientras que durante el ciclo 2016 el rendimiento de ambas variedades fluctuó entre 3200 y $3687 \mathrm{~kg} \mathrm{ha}^{-1}$. En este sitio se observaron resultados similares a los obtenidos en otros estudios con frijol, donde se dificultó la obtención de diferencias estadísticas entre niveles de aplicación del agua de riego. Con los resultados obtenidos en los dos sitios se comprobó que el manejo eficiente del agua de riego debe ajustarse con base en las condiciones meteorológicas del sitio de

Cuadro 2. Lámina de riego en función de los niveles de humedad y variables meteorológicas registradas en los sitios y ciclos de evaluación.

\begin{tabular}{|c|c|c|c|c|c|c|}
\hline \multirow{3}{*}{ Sitio } & & hum & & \multirow{3}{*}{$\mathrm{T}_{\text {máx }}\left({ }^{\circ} \mathrm{C}\right)$} & \multirow{3}{*}{$\mathrm{T}_{\min }\left({ }^{\circ} \mathrm{C}\right)$} & \multirow{3}{*}{$\mathrm{Pp}(\mathrm{mm})$} \\
\hline & 100 & 80 & 60 & & & \\
\hline & \multicolumn{3}{|c|}{$L r_{a}(m m)$} & & & \\
\hline Durango (2015) & 544 & 204 & 156 & 27.6 & 13.2 & 221 \\
\hline Canatlán (2015) & 404 & 270 & 174 & 27.3 & 11.5 & 227 \\
\hline Durango (2016) & 209 & 181 & 83 & 26.8 & 14.2 & 357 \\
\hline
\end{tabular}

$T_{\text {máx }}$ : temperatura máxima, $T_{\text {min }}$ : temperatura mínima, Pp: precipitación, $L r_{a}$ : lámina de riego aplicada.

Cuadro 3. Fenología y rendimiento registrado en dos variedades de frijol evaluadas bajo tres niveles de humedad.

\begin{tabular}{|c|c|c|c|c|c|c|c|c|c|}
\hline \multirow{2}{*}{ Variedad } & \multicolumn{3}{|c|}{ Durango (2015) } & \multicolumn{3}{|c|}{ Canatlán (2015) } & \multicolumn{3}{|c|}{ Durango (2016) } \\
\hline & $\mathrm{df}$ & $\mathrm{dmf}$ & $\mathrm{Rd}$ & $\mathrm{df}$ & $\mathrm{dmf}$ & $\mathrm{Rd}$ & $\mathrm{df}$ & $\mathrm{dmf}$ & $\mathrm{Rd}$ \\
\hline$L r+P p$ & \multicolumn{3}{|c|}{$100 \%(765 \mathrm{~mm})$} & \multicolumn{3}{|c|}{$100 \%(631 \mathrm{~mm})$} & \multicolumn{3}{|c|}{$100 \%(566 \mathrm{~mm})$} \\
\hline P. Saltillo & $43_{(448)}$ & $103_{(1,013)}$ & 3129 & $45_{(459)}$ & $101_{(950)}$ & $3344^{a}$ & $43_{(448)}$ & $95_{(917)}$ & 3576 \\
\hline P. Centauro & $41_{(427)}$ & $97_{(967)}$ & 2546 & $40_{(406)}$ & $99_{(937)}$ & $3157^{a}$ & $41_{(430)}$ & $92_{(893)}$ & 3548 \\
\hline$L r+P p$ & \multicolumn{3}{|c|}{$80 \%$ (425 mm) } & \multicolumn{3}{|c|}{$80 \%(497 \mathrm{~mm})$} & \multicolumn{3}{|c|}{$80 \%(538 \mathrm{~mm})$} \\
\hline P. Saltillo & $42_{(438)}$ & $102_{(1,007)}$ & 3380 & $45_{(459)}$ & $99_{(937)}$ & $1946^{b}$ & $42_{(440)}$ & $94_{(909)}$ & 3466 \\
\hline P. Centauro & $41_{(427)}$ & $96_{(958)}$ & 2890 & $41_{(416)}$ & $98_{(930)}$ & $2190^{b}$ & $41_{(430)}$ & $93_{(900)}$ & 3687 \\
\hline$L r+P p$ & \multicolumn{3}{|c|}{$60 \%(377 \mathrm{~mm})$} & \multicolumn{3}{|c|}{$60 \%(401 \mathrm{~mm})$} & \multicolumn{3}{|c|}{$60 \%(440 \mathrm{~mm})$} \\
\hline P. Saltillo & $43_{(448)}$ & $103_{(1,013)}$ & 2665 & $44_{(447)}$ & $100_{(943)}$ & $1645^{\circ}$ & $43_{(448)}$ & $93_{(900)}$ & 3292 \\
\hline P. Centauro & $41_{(427)}$ & $97_{(967)}$ & 2686 & $41_{(416)}$ & $97_{(923)}$ & $1705^{c}$ & $41_{(430)}$ & $92_{(893)}$ & 3200 \\
\hline Media & $42_{(438)}$ & $99_{(986)}$ & 2883 & $43_{(436)}$ & $99_{(937)}$ & 2331 & $42_{(440)}$ & $93_{(900)}$ & 3461 \\
\hline
\end{tabular}

Letras en cada columna representan diferencias significativas entre variedades y $L r+P p$ (Tukey, $P \leq 0.05)$. Las columnas sin letras indican que no hay diferencias significativas. df: días a floración, dmf: días a madurez fisiológica (el subíndice representa la acumulación de grados día, `D), Rd: rendimiento $\mathrm{kg} \mathrm{ha}^{-1}, \mathrm{Lr}+\mathrm{Pp}$ : acumulación total de lámina de riego más la precipitación. 
siembra y el tipo de suelo que se utiliza en la producción de frijol.

El ambiente con mayor productividad fue Durango (2016), con una Lr total entre 538 mm (RD $80 \%$ ) y 566 mm (RT $100 \%$ ). El siguiente nivel de producción se observó en Canatlán, con una Lr total aplicada de $631 \mathrm{~mm}$ para ambas variedades. En este último sitio se registró el rendimiento promedio más bajo, independientemente de las variedades de frijol, debido a la combinación del bajo nivel de aplicación de agua (401 mm) y tipo de suelo (arena franca); además, se registraron temperaturas altas $\left(>28^{\circ} \mathrm{C}\right)$ en las etapas vegetativas y temperaturas bajas $\left(<11.5^{\circ} \mathrm{C}\right)$ en las etapas reproductivas, lo que influyó en la respuesta del rendimiento del frijol.

\section{Fenología y acumulación de grados día $\left({ }^{\circ} D\right)$}

En Durango (2015), ambas variedades mostraron similitud en los días para emergencia y ${ }^{\circ} D\left(8_{84} d d s_{{ }_{0}}\right)$, exposición de hojas primarias $\left(12_{124} \mathrm{dds}_{{ }_{0} \mathrm{D}}\right)$ y primera hoja trifoliolada $\left(15_{153} \mathrm{dds}_{{ }_{0} \mathrm{D}}\right)$. Comparada con Pinto Centauro, la variedad Pinto Saltillo requirió menor cantidad de días a la aparición de la tercera hoja trifoliolada ( $21_{211}$ vs. $23_{228} \mathrm{dds}_{\mathrm{o}_{\mathrm{D}}}$ ) y demoró en prefloración $\left(41_{424} v s .39_{406} \mathrm{dds}_{0_{0}}\right)$, floración $\left(43_{445}\right.$ vs. $41_{424}$ dds $\left._{\text {oD }}\right)$, formación de vainas $\left(56_{582}\right.$ vs. $53_{551}$ dds $\left.{ }_{{ }_{0} \mathrm{D}}\right)$, llenado de grano $\left(60_{636}\right.$ vs. $\left.57_{602} d d s_{\circ \mathrm{OD}}\right)$ y madurez fisiológica $\left(103_{1013}\right.$ vs. $\left.97_{964} \mathrm{dds}_{\mathrm{o}_{\mathrm{D}}}\right)$ (Figura 1).

En Canatlán (2015), la variedad Pinto Centauro mostró una emergencia tardía $\left(8_{77} \mathrm{dds}_{{ }_{0}}\right)$ con respecto a Pinto Saltillo $\left(7_{71} \mathrm{dds}_{{ }_{0 D}}\right)$ (Figura 2). Ambas variedades mostraron igualdad en el desarrollo de las hojas primarias $\left(12_{115} \mathrm{dds}_{{ }_{\text {oD }}}\right)$ y aparición de la primera hoja trifoliolada $\left(15_{142} \mathrm{dds}_{{ }_{0} \mathrm{D}}\right)$. En Pinto Centauro se registró una demora en la aparición de la tercera hoja trifoliolada $\left(21_{199} \mathrm{dds}_{\mathrm{o}_{\mathrm{D}}}\right)$, debido a que Pinto Saltillo redujo los valores para esta variable $\left(19_{178} \mathrm{dds}_{\text {oD }}\right)$. La variedad Pinto Saltillo presentó demora en prefloración, comparada con Pinto Centauro ( $43_{432}$ vs. $\left.39_{394} d d s_{o_{0}}\right)$, floración $\left(45_{455}\right.$ vs. $\left.41_{413} \mathrm{dds}_{{ }_{0 \mathrm{D}}}\right)$, formación de vainas $\left(54_{551}\right.$ vs. $51_{526}$ dds $\left._{\circ \mathrm{o}}\right)$, llenado de grano $\left(59_{616}\right.$ vs. $\left.57_{586} \mathrm{dds}_{\circ \mathrm{D}}\right)$ y madurez fisiológica $\left(100_{943}\right.$ vs. $\left.98_{930} \mathrm{dds}_{{ }^{\circ}}\right)$.

En promedio, independientemente de la variedad y $L r$, en Canatlán (2015) se redujeron las unidades térmicas con respecto a Durango (2015) con ( $97_{923}$ vs. $96_{958} \mathrm{dds}_{{ }_{0 \mathrm{D}}}$ ) debido a la temperatura ambiental más alta (28 a 30.7 ${ }^{\circ} \mathrm{C}$ ) en la etapa vegetativa y reproductiva (meses de julio a septiembre). Después se registraron períodos con $T_{\text {min }}$ ambiental de $14.1^{\circ} \mathrm{C}$ durante el llenado de grano (tercera semana de septiembre) (Figura 3). Se observa que la temperatura alta, combinada con el estrés hídrico, es un factor a considerar para definir la duración de las etapas fenológicas del frijol (Rosales-Serna et al., 2001).
En Durango (2016), la variedad Pinto Centauro mostró ser tardía en la emergencia $\left(9_{100} \mathrm{dds}_{\text {oD }}\right)$, con respecto a Pinto Saltillo $\left(7_{78} d d_{s_{0}}\right)$ (Figura 4); además, Pinto Centauro registró una demora para el despliegue de las hojas primarias $\left(12_{133} \mathrm{dds}_{{ }_{0} \mathrm{D}}\right)$, primera hoja trifoliolada $\left(15_{169}\right.$ $\mathrm{dds}_{\text {oD }}$ ) y tercera hoja trifoliolada $\left(25_{267} \mathrm{dds}_{\text {oD }_{\mathrm{D}}}\right)$, debido a que Pinto Saltillo redujo los valores para estas variables $\left(10_{110}\right.$ $\left.\mathrm{dds}_{{ }_{0}} ; 13_{145} \mathrm{dds}_{o_{0}} ; 22_{241} \mathrm{dds}_{{ }_{0} \mathrm{D}}\right)$; después, la variedad Pinto Saltillo presentó una demora para la prefloración $\left(41_{424}\right.$ vs. $39_{412}$ dds $\left._{0_{0}}\right)$, floración $\left(43_{445}\right.$ vs. $41_{430}$ dds $\left.{ }_{{ }_{0} D}\right)$, formación de vainas $\left(55_{548}\right.$ vs. $52_{525}$ dds $\left.{ }_{\circ 0}\right)$, llenado de grano $\left(69_{680}\right.$ vs. $\left.66_{653} \mathrm{dds}_{\mathrm{o}_{\mathrm{D}}}\right)$ y madurez fisiológica $\left(94_{909} \mathrm{vs.} 92_{895} \mathrm{dds_{o_{D } }}\right.$ ).

La demora inicial en la fenología de la variedad de frijol Pinto Centauro fue consecuencia de las características genéticas de la variedad, a pesar de la utilización de semillas con mayor tamaño (34 g por 100 semillas), en comparación con Pinto Saltillo (31 g por 100 semillas) (Rosales et al., 2012), lo que retrasó la imbibición, germinación y emergencia. Se observó relación estrecha entre el registro de la fenología con base en el número de dds y la cantidad de ${ }^{\circ} D$ acumulados. La duración de las etapas fenológicas se redujo en Durango (2016) debido a un incremento en la $T_{\text {min }}$ promedio $\left(14.2^{\circ} \mathrm{C}\right)$, con ello se pone de manifiesto que la duración de las etapas fenológicas está en función del comportamiento térmico durante el período de crecimiento de la especie, pero será constante en términos de ${ }^{\circ} \mathrm{D}$. Lo anterior, aceleró el desarrollo y redujo el ciclo biológico de las dos variedades bajo estudio. La $T_{\text {máx }} y T_{\text {min }}$ del ambiente influenciaron en la respuesta del frijol en todos los tratamientos con niveles de humedad, por lo que este factor debe considerarse al calendarizar el riego.

\section{Productividad del agua (PA)}

Se observó tendencia a reducir la PA cuando se incrementó la lámina de riego aplicada $\left(L_{\mathrm{a}}\right)$ a las variedades de frijol (Figura 5). El nivel más alto de PA (7.3 a $8.0 \mathrm{~kg} \mathrm{ha}^{-1}$ $\mathrm{mm}^{-1}$ ) en ambas variedades se registró con una $\mathrm{Lr}_{a}$ entre 425 y $460 \mathrm{~mm}$. En las $L_{2}$ que sobrepasaron los $750 \mathrm{~mm}$ se registró un nivel bajo de PA (3.3 $\left.\mathrm{kg} \mathrm{ha}^{-1} \mathrm{~mm}^{-1}\right)$ debido al exceso de humedad (por la combinación de $L r+P p$ ) con respecto al nivel recomendado para frijol, que oscila de 400 a 631 mm (Rosales-Serna et al., 2016; Villordo-Pineda et al., 2015).

Se ha observado sensibilidad del frijol en ambientes con humedad excesiva y con deficiencia en el control de la aplicación del agua de riego debido al estrés por oxígeno (hipoxia) y decremento en la fotosíntesis y conductancia estomática (Caudle y Maricle, 2012), lo cual afecta la producción de biomasa y el rendimiento de grano (Polón-Pérez et al., 2017). Pedroza-Sandoval et al. (2016) 


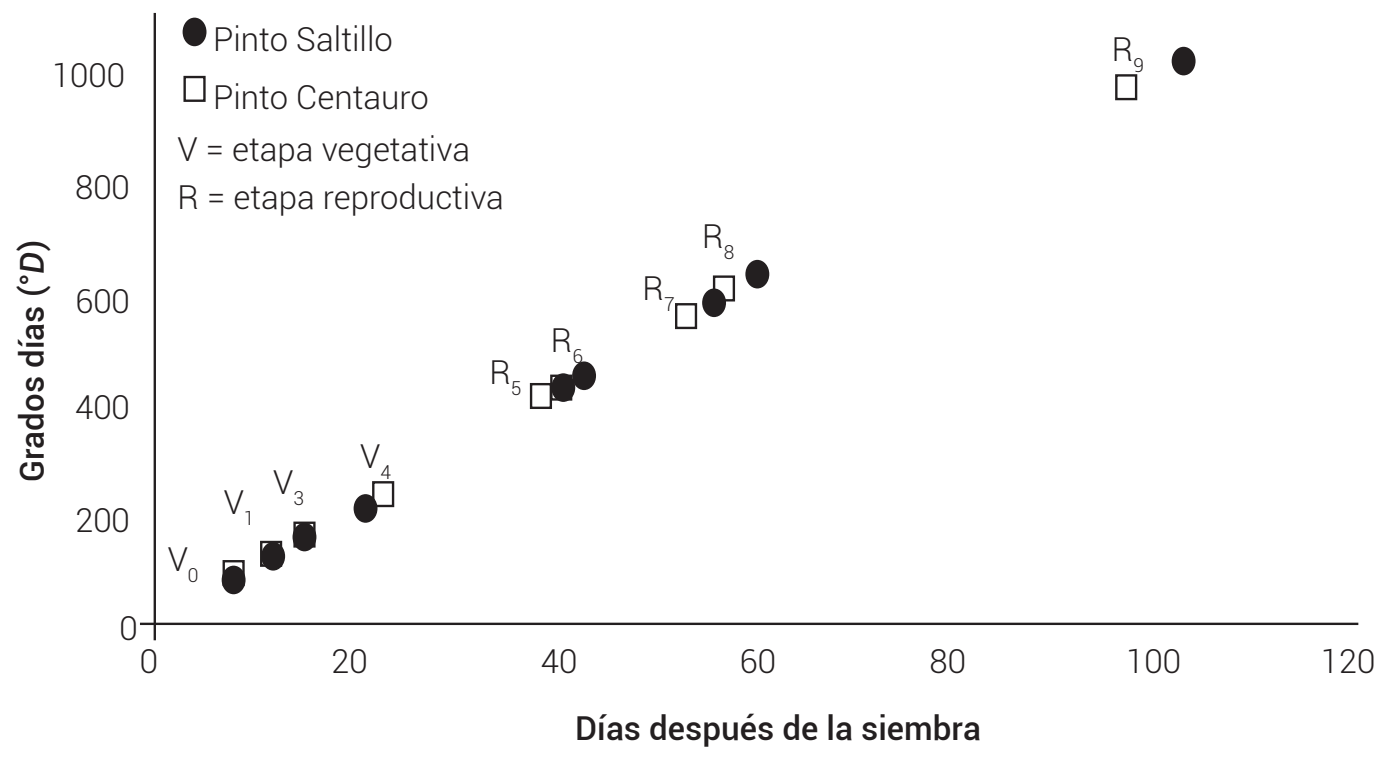

Figura 1. Relación entre el número de días para alcanzar cada etapa fenológica y la cantidad de grados día $\left({ }^{\circ} D\right)$ acumulados en dos variedades de frijol cultivadas en Durango (2015).

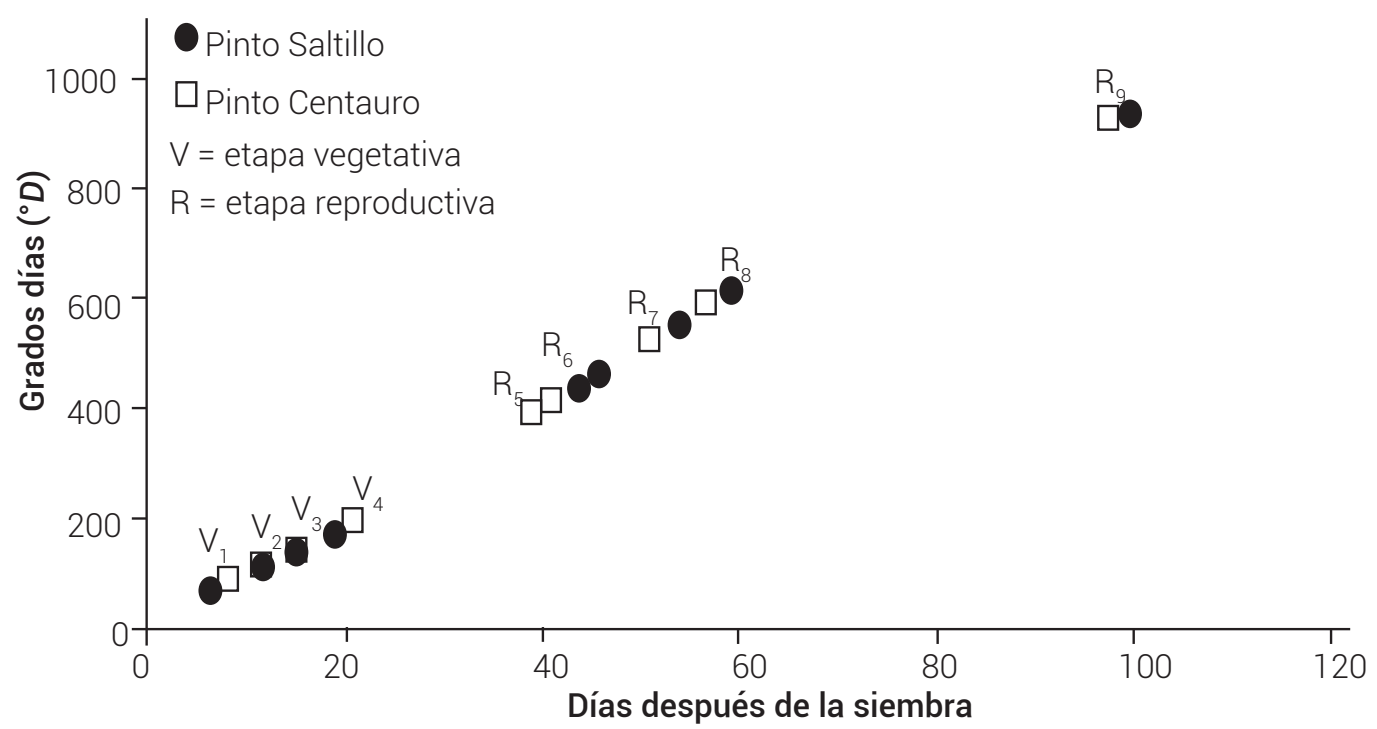

Figura 2. Relación entre el número de días para alcanzar una etapa fenológica y la cantidad de grados día $\left({ }^{\circ} D\right)$ acumulados en dos variedades de frijol en Canatlán (2015).

obtuvieron resultados similares en tres variedades de frijol, las cuales tuvieron un comportamiento similar en la actividad fotosintética al pasar de una condición hídrica a otra, aunque hubo una tendencia de mayor estabilidad en las variedades Pinto Centauro y Pinto Saltillo, las cuales varían en su actividad fotosintética; además, observaron valores bajos de productividad para ambas variedades con $L r_{a}$ de 401 y $497 \mathrm{~mm}$ en el sitio de Canatlán, donde el suelo es arena franca y se registraron $T_{\text {mạ́x }}$ altas (28 a $30^{\circ} \mathrm{C}$ ) durante la etapa vegetativa y reproductiva (Figura 3). Los valores altos de temperatura (Figura 4) incrementaron la demanda ambiental y ésto, combinado con el reducido 

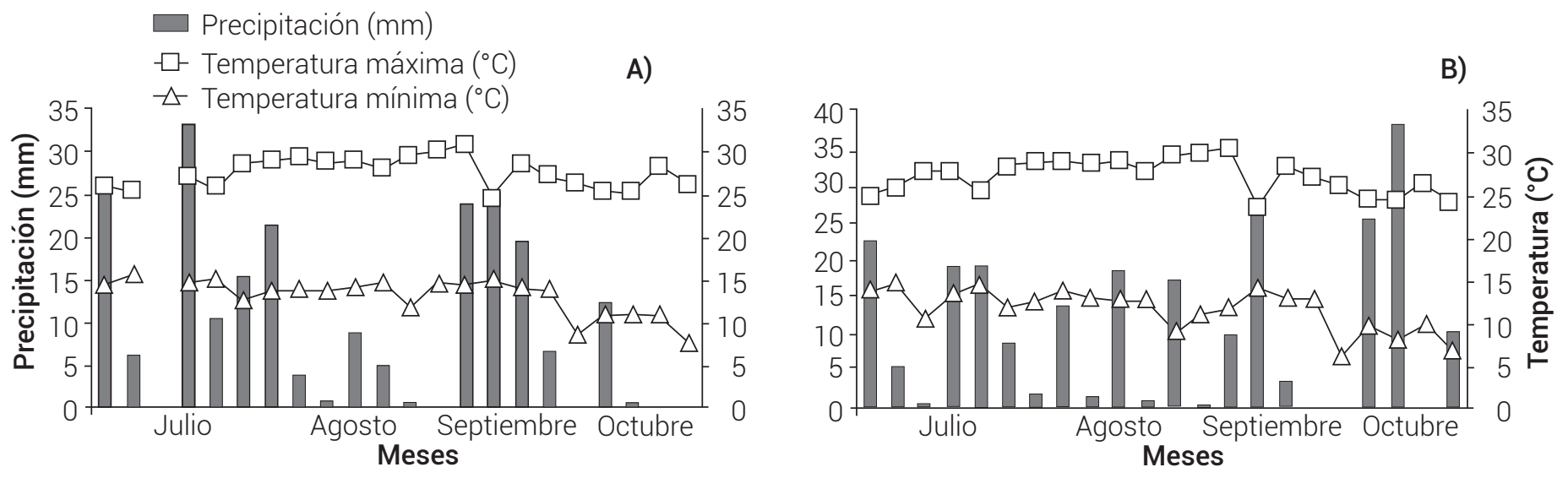

Figura 3. Valores diarios de precipitación, $T_{\text {máx }}$ y $T_{\text {min }}$ registradas en Durango (A) y Canatlán (B) en el año 2015.

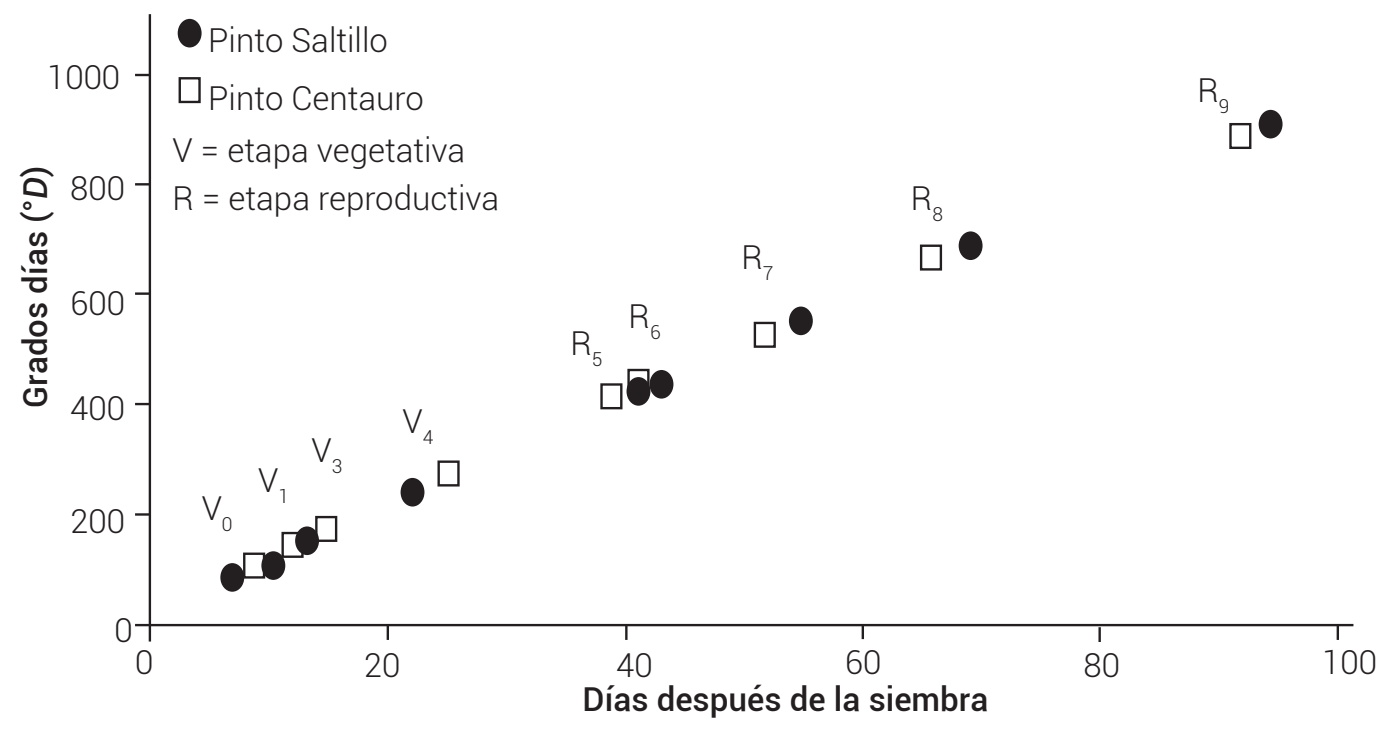

Figura 4. Relación entre el número de días para alcanzar una etapa fenológica y la cantidad de grados día acumulados $\left({ }^{\circ} D\right)$ en dos variedades de frijol en Durango (2016).

suministro de agua en los tratamientos de RD $80 \%$ y RD $60 \%$, redujo el rendimiento.

La PA en las variedades de frijol, evaluadas a través de sitios y tratamientos de humedad, fluctuó entre 3.3 y $8.0 \mathrm{~kg} \mathrm{ha}^{-1} \mathrm{~mm}^{-1}$. Los valores resultaron inferiores a los observados en otros estudios con frijol, en los que se determinaron valores entre 4.6 y $10 \mathrm{~kg} \mathrm{ha}^{-1} \mathrm{~mm}^{-1}$ (Beebe et al., 2013). La fluctuación en la PA se relacionó directamente con el tipo de suelo, el sitio de siembra y la $\mathrm{Lr}_{\mathrm{a}}$. La aplicación apropiada del agua de riego en Durango duplicó el rendimiento y redujo en 51 \% (2015) y 22 \% (2016) la cantidad de agua utilizada en la producción de frijol con el nivel de RD $80 \%$, sin disminuir significativamente el rendimiento. En Canatlán, la disminución de $21 \%$ (RD $80 \%$ ) y $36 \%$ (RD $60 \%$ ) del agua aplicada redujo significativamente el rendimiento de grano, debido al tipo de suelo y $T_{\text {máx }} y T_{\text {min }}$ registradas durante el ciclo de cultivo, por lo que, ante este tipo de escenarios de manejo y condiciones de producción, la tecnificación de la superficie cultivada con frijol puede ser lo más viable en la medida de las condiciones y posibilidades de los productores (Ríos et al., 2017). 


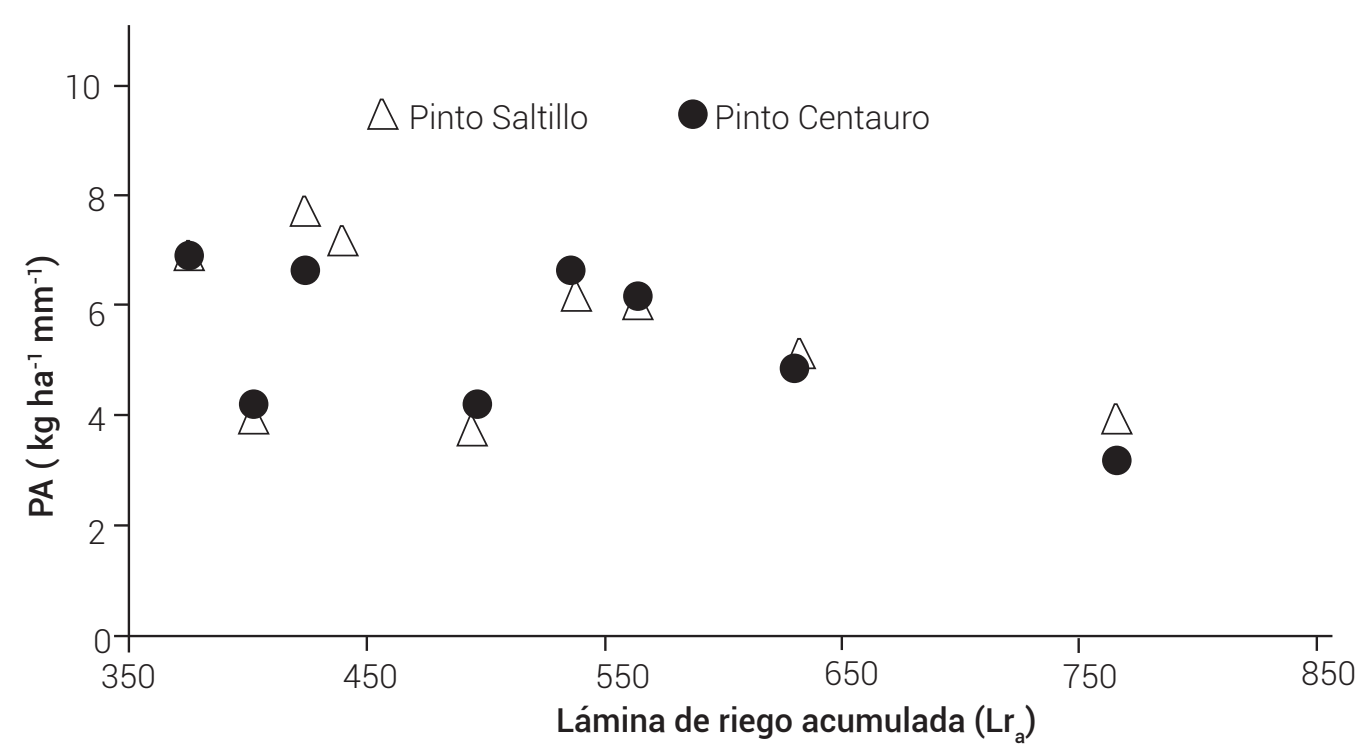

Figura 5. Relación promedio entre la $\mathrm{Lr}_{\mathrm{a}}$ y la PA en dos variedades de frijol cultivadas en tres sitios y tres niveles de humedad.

\section{CONCLUSIONES}

Las condiciones favorables de humedad proporcionadas con los niveles de RT $100 \%$ y RD $80 \%$ favorecieron un incremento en el rendimiento de grano de las variedades de frijol Pinto Saltillo y Pinto Centauro. La respuesta fenológica estuvo ligada al suministro oportuno y preciso del riego, de acuerdo con cada nivel de humedad, lo cual está en función de la variabilidad climática del sitio y las condiciones edáficas. Pinto Centauro mostró mayor precocidad en los sitios y ciclos de evaluación con respecto a Pinto Saltillo; por lo tanto, se requiere la generación de estudios a nivel local, pues existen pocos sobre la temática, y los requerimientos hídricos varían de acuerdo con el área agrícola, clima, manejo agronómico, variedad y fenología del genotipo. Las variedades de frijol Pinto Saltillo y Pinto Centauro mostraron valores altos para el rendimiento de grano con los niveles de humedad de RD 80 \% y RT 100 $\%$, por lo cual, esta leguminosa es una opción productiva para condiciones de riego, donde se pueden obtener rendimientos comercialmente viables comparados con los obtenidos bajo condiciones de temporal.

\section{AGRADECIMIENTOS}

El presente estudio se desarrolló como parte del proyecto: Parametrización de modelos para el pronóstico del riego en tiempo real a través de un portal web y dispositivos móviles, financiado con Fondos Fiscales INIFAP (clave SIGI: 1123333087).

\section{BIBLIOGRAFÍA}

Arellano-Arciniega S., E. S. Osuna-Ceja, M. A. Martínez-Gamiño y L. ReyesMuro (2015) Rendimiento de frijol fertilizado con estiércol bovino en condiciones de secano. Revista Fitotecnia Mexicana 38:313318, https://doi.org/10.35196/rfm.2015.3.313

Baez-González A. D., J. R. Kiniry, J. S. Padilla R., G. Medina G., J. L. Ramos G. and E. S. Osuna C. (2015) Parametrization of ALMANAC crop simulation model for non-irrigated dry bean in semi-arid temperate areas in Mexico. Interciencia 40:185-189.

Beebe S. E., I. M. Rao, M. W. Blair and J. A. Acosta-Gallegos (2013) Phenotyping common beans for adaptation to drought. Frontiers in Physiology 4:35, http://doi.org/10.3389/fphys.2013.00035

Borja-Bravo M., E. S. Osuna-Ceja, S. Arellano-Arciniega, R. V. García-Hernández y M. Á. Martínez-Gamiño (2018) Competitividad y eficiencia en la producción de frijol en condiciones de temporal con tecnología tradicional y recomendada. Revista Fitotecnia Mexicana 41:443-450, https://doi.org/10.35196/rfm.2018.4.443-450

Caudle K. L. and B. R. Maricle (2012) Effects of flooding on photosynthesis, chlorophyll fluorescence, and oxygen stress in plants of varying flooding tolerance. Transactions of the Kansas Academy of Science 115:5-18, https://doi.org/10.1660/062.115.0102

de Medeiros G. A., L. A. Daniel and F. H. Fengler (2016) Growth, development, and water consumption of irrigated bean crop related to growing degree-days on different soil tillage systems in southeast Brazil. International Journal of Agronomy 2016:8065985, https://doi. org/10.1155/2016/8065985

de Oliveira E. C., J. M. N. da Costa, T. J. de Paula Jr., W. P. M. Ferreira, F. B. Justino and L. O. Neves (2012) The performance of the CROPGRO model for bean (Phaseolus vulgaris L.) yield simulation. Acta Scientiarum, Agronomy 34:239-246, https://doi.org/10.4025/ actasciagron.v34i3.13424

Flores-Gallardo H., W. Ojeda-Bustamante, H. Flores-Magdaleno, E. MejíaSáenz y E. Sifuentes-Ibarra (2012) Grados día y la programación integral del riego en el cultivo de papa. Terra Latinoamericana 30:59-67.

Flores-Magdaleno H., H. Flores-Gallardo y W. Ojeda-Bustamante (2014) Predicción fenológica del cultivo de papa mediante tiempo térmico. Revista Fitotecnia Mexicana 37:149-157, https://doi org/10.35196/rfm.2014.2.149

García E. (1987) Modificaciones al Sistema de Clasificación Climática de Köppen (Para Adaptarlo a las Condiciones de la República Mexicana). 4a edición. Instituto de Geografía, Universidad Nacional Autónoma de México. México, D. F. 217 p. 
Guzmán M. S. H., J. A. Acosta G., M. Á. Álvarez-Muñoz, S. García-Delgado y G. Loarca-Piña (2002) Calidad alimentaria y potencial nutracéutico del frijol (Phaseolus vulgaris L.). Agricultura Técnica en México 28:159-173.

Hari Krishna B., A. Mani, M. Uma Devi and V. Ramulu (2014) Water productivity of agricultural crops in upper Manair catchment International Journal of Innovative Research and Development 3:377-382

INIFAP, Instituto Nacional de Investigaciones Forestales, Agrícolas y Pecuarias (2017) Red Nacional de Estaciones Agrometeorológicas Automatizadas. Instituto Nacional de Investigaciones Forestales, Agrícolas y Pecuarias. Ciudad de México. https:// datos.gob.mx/busca/dataset/red-nacional-de-estacionesagrometeorologicas-automatizadas-inifap/ (Noviembre 2021).

Jenni S., G. Bourgeois, H. Laurence, G. Roy and N. Tremblay (2000) Improving the prediction of processing bean maturity based on the growing-degree day approach. HortScience 35:1234-1237, https://doi.org/10.21273/HORTSCI.35.7.1234

Ojeda-Bustamante W., E. Sifuentes-Ibarra, D. C. Slack and M. Carrillo (2004) Generalization of irrigation scheduling parameters using the growing degree days concept: application to a potato crop Irrigation and Drainage 53:251-261, https://doi.org/10.1002/ ird. 134

Ojeda-Bustamante W., E. Sifuentes-Ibarra y H. Unland-Weiss (2006) Programación integral del riego en maíz en el norte de Sinaloa, México. Agrociencia 40:13-25.

Pedroza-Sandoval A., R. Trejo-Calzada, I. Sánchez-Cohen, J. A. SamaniegoGaxiola y L. G. Yáñez-Chávez (2016) Evaluación de tres variedades de frijol pinto bajo riego y sequía en Durango, México. Agronomía Mesoamericana 27:167-176, https://doi. org/10.15517/am.v27i1.21896

Polón-Pérez R., M. Ruiz-Sánchez, A. Miranda-Caballero y M. A. RamírezArrebato (2017) Efectos del estrés hídrico sobre el rendimiento de los granos del frijol (Phaseolus vulgaris L.). Revista Ciencias Técnicas Agropecuarias 26:66-70.

Ríos F. J. L., M. Torres Mo., M. A. Torres M. y J. E. Cantú B. (2017) Eficiencia y productividad del cultivo de frijol en un sistema de riego por bombeo en Zacatecas, México. CIENCIA ergo-sum 24:152-163, https://doi.org/10.30878/ces.v24n2a7
Ríos-Uglade M. C., R. Reynoso-Camacho, I. Torres-Pacheco, J. A. AcostaGallegos, A. C. Palomino-Salinas, M. Ramos-Gómez, ... and S. H. Guzmán-Maldonado (2006) Effect of common bean (Phaseolus vulgaris) consumption on colon cancer in Spargue-Dawley rats. Annual Report of the Bean Improvement Cooperative 49:123 124.

Rosales S. R., F. J. Ibarra P. y E. I. Cuéllar R. (2012) Pinto Centauro, nueva de frijol para el estado de Durango. Revista Mexicana de Ciencias Agrícolas 3:1467-1474, https://doi.org/10.29312/remexca. v3i7.1354

Rosales-Serna R., R. Ochoa M. y J. A. Acosta G. (2001) Fenología y rendimiento del frijol en el Altiplano de México y su respuesta al fotoperiodo. Agrociencia 35:513-523.

Rosales-Serna R., J. Kohashi-Shibata, J. A. Acosta-Gallegos, C. Trejo-López, J. Ortiz-Cereceres and J. D. Kelly (2004) Biomass distribution, maturity acceleration and yield in drought-stressed common bean cultivars. Field Crops Research 85:203-211, https://doi org/10.1016/S0378-4290(03)00161-8

Rosales-Serna R., H. Flores-Gallardo and J. C. López-González (2016) Water regime effects on phenology and seed yield of common bean in Durango, México. Annual Report of the Bean Improvement Cooperative 59:241-242.

Rosales-Serna R., H. Flores-Gallardo, C. A. Nava-Berumen y A. I. Ortiz-Sánchez (2019) Rendimiento y calidad de grano en líneas mejoradas de frijol cultivadas bajo riego en Durango, México. Revista Fitotecnia Mexicana 42:39-46, https://doi.org/10.35196/ rfm.2019.1.39-46

Sanchez-Valdez I., J. A. Acosta-Gallegos, F. J. Ibarra-Pérez, R. Rosales-Serna and S. P. Singh (2004) Registration of 'Pinto Saltillo' common bean. Crop Science 44:1865-1866, https://doi.org/10.2135/ cropsci2004.1865a

SAS Institute (2013) Base SAS ${ }^{\circledast} 9.4$ Procedures Guide: Statistical Procedures, 2nd edition. SAS Institute Inc. Cary, North Carolina, USA. $558 \mathrm{p}$.

Villordo-Pineda E., M. M. González-Chavira, P. Giraldo-Carbajo, J. A. Acosta-Gallegos and J. Caballero-Pérez (2015) Identification of novel drought-tolerant-associated SNPs in common bean (Phaseolus vulgaris). Frontiers in Plant Science 6:546, https:// doi.org/10.3389/fpls.2015.00546 
